\title{
The basic science behind overactive bladder: introduction
}

\section{Jerzy Gajewski, MD, FRCSC}

Department of Urology, Dalhousie University, Halifax, NS
Cite as: Can Urol Assoc J 2011;5(5Suppl2):S125; D01:10.5489/cuaj.11179

$\mathrm{T}$ he objective of this section of the supplement is to discuss the multifactorial pathophysiology of the overactive bladder syndrome $(\mathrm{OAB})$ and translate this knowledge into the clinical environment. There are two separate articles on the pathophysiology, with Dr. Christopher Chapple focusing on the role of central neurogenic pathways and Dr. Pradeep Tyagi discussing the local pathways (neurogenic, myogenic and urothelial). The new look into this area of human bladder innervations brings new ideas of how to approach voiding dysfunction and particularly OAB. This is important to finding other mechanisms of intervention in $\mathrm{OAB}$; exploring central nervous system (CNS) and specific neurogenic pathways opens up new areas of research and intervention.

In recognition of the multiple pathways of bladder dysfunction, medical science has identified many potential tar- gets for pharmacologic intervention. The third article of this section by Dr. Karl-Erik Andersson is devoted to research into the future pharmacological compounds to be tested in $\mathrm{OAB}$. In the subsequent sections of this publication, clinical aspects of therapeutic efficacy of current and emerging treatment agents will be discussed in even greater detail.

Competing interests: None declared.

This paper has been peer-reviewed.

Correspondence: Dr. Jerzy B. Gajewski, Professor of Urology \& Pharmacology, Department of Urology, Dalhousie University, QEll Health Science Centre, HI Site, 1225B-1796 Summer St., Halifax, NS B3H 3A7; fax: 902-473-8568; igajew@dal.ca 